Proceedings

\title{
Current promising antibiotics and future approaches in combating carbapenemase-producing Enterobacteriaceae ${ }^{\dagger}$
}

\author{
Corneliu Ovidiu Vrancianu ${ }^{1,2}$, Elena Georgiana Dobre ${ }^{1}$, Irina Gheorghe ${ }^{1,2, *}$, Ilda Barbu ${ }^{1,2}$, Roxana \\ Elena Cristian $^{3}$ and Mariana Carmen Chifiriuc ${ }^{1,2}$ \\ ${ }^{1}$ University of Bucharest, Faculty of Biology, Microbiology Immunology Department; \\ ovidiu.vrancianu@yahoo.com (C.O.V.); dobregeorgiana_95@yahoo.com (E.G.D.); ilda.czobor@yahoo.com \\ (I.B.C.); carmen.chifiriuc@bio.unibuc.ro (M.C.C.) \\ 2 The Research Institute of the University of Bucharest, Bucharest, Romania; \\ ${ }^{3}$ Department of Biochemistry and Molecular Biology, Faculty of Biology, University of Bucharest, Bucharest, \\ Romania; roxana.cristian95@yahoo.com (R.E.C.); \\ * Correspondence: iryna_84@yahoo.com (I.G.) \\ + Presented at the 1st International Electronic Conference on Microbiology, 2-30 November 2020; Available \\ online: https://ecm2020.sciforum.net/
}

Published: 2 November 2020

\begin{abstract}
Carbapenem-resistant (CR) Gram-negative bacilli, including Enterobacteriaceae and the non-fermenters, represent the most notorious pathogens due to the high incidence of morbidity and mortality, especially in immunocompromised patients in intensive care units. Carbapenem resistance is mainly associated with the production of carbapenemases, which are $\beta$-lactamases belonging to different Ambler classes (A, B, D) that can be encoded by both chromosomal and plasmid-mediated genes. These enzymes represent the most potent $\beta$-lactamases, hydrolyzing a wide variety of $\beta$-lactams, including carbapenems, cephalosporins, penicillin, and aztreonam. The major issues associated with carbapenemases production are both clinical, posing significant challenges in the treatment of healthcare-associated infections by compromising the activity of the last-resort antibiotics, and epidemiological, due to their dissemination across almost all geographic regions. An important advancement is a handful of recently launched new antibiotics targeting some of the current most problematic Gram-negative pathogens, namely carbapenem-resistant Enterobacteriaceae (CRE). The most appropriate antimicrobial therapy to treat CRE infections is still controversial. Combination therapy is preferred over monotherapy due to its broad-spectrum coverage, synergic activity, and low probability of selecting for resistance. In this mini-review, current and future promising antibiotics that are currently under investigation for winning the war against the emerging CRE are discussed.
\end{abstract}

Keywords: carbapenem-resistant Enterobacteriaceae (CRE), carbapenamases; multi-drug resistance; novel antibiotics

\section{Introduction}

In 2017, World Health Organization (WHO) published a list of resistant bacteria against which there is an urgent need to develop new antibiotics [1]. Critically priority bacteria include carbapenemresistant Enterobacteriaceae (CRE). These bacteria are among the most common pathogens associated with severe infections such as sepsis, pneumonia, urinary tract, and intra-abdominal infections. Consequently, CREs have a major impact, both clinically and economically [2]. Initially, Enterobacteriaceae posed a threat to public health because of their ability to resist the action of betalactam antibiotics by producing broad-spectrum beta-lactamases. This situation has led to the use of 
carbapenems as first-line drugs [3]. The use of carbapenems as a treatment for resistant bacteria has led to CRE's emergence over time. In response to the CRE threat, efforts have been made to reduce the spread and prevent the development of resistance from reducing nosocomial infections associated with CRE by up to $60 \%$ by the end of 2020 [4]. The American Society of Infectious Diseases has launched the "10x20" campaign to develop 10 new antibiotics by the end of 2020, with two such antibiotics already receiving FDA approval [5].

For challenging the Gram-negative resistant bacteria, polymyxins and tigecycline were considered an option, but resistance to these antibiotics is increasing alarmingly [6]. Also, phosphomycin and aminoglycosides are used occasionally [7]. Carbapenems still play an important role in treating CRE either in high doses in combination with other active agents or dual therapy. Old antibiotics such as minocycline, doxycycline, sulfamethoxazole (SXT), and chloramphenicol may be effective against certain CRE isolates [8,9]. In response to increasing resistance, several new antibiotics have been developed that target KPC-producing Enterobacteriaceae and multiple-drug resistant (MDR) P. aeruginosa. Other antibiotics in the final stages of development target pathogens that produce metallo-beta-lactamases (MBL) and MDR A. baumannii [10]. Currently, ceftazidimeavibactam, ceftolozane-tazobactam, meropenem-vaborbactam, and eravacycline have been included in clinical therapy in America and Europe, while plazomicin has received FDA approval [10].

\section{Current Promising Antibiotics in Treating CRE Infections}

Selecting an antimicrobial compound for carbapenem-resistant (CR) Gram-negative infections is almost always challenging, though the degree of difficulty varies depending on the specific clinical scenario. The major issues associated with carbapenemase production are clinical due to compromising the last-resort antibiotics activity used for treating serious infections and epidemiological due to their dissemination into various bacteria across almost all geographic regions. An important advancement is the recently launched new antibiotics targeting some of the current most problematic Gram-negative pathogens, namely carbapenem-resistant Enterobacteriaceae (CRE). Recently, ceftazidime-avibactam and meropenem-vaborbactam for CRE and ceftolozane-tazobactam for carbapenem-resistant P. aeruginosa infections have become important treatment options in countries where these agents have become available for clinical use [11]. Other new agents like plazomicin, recently approved by FDA, eravacycline, potent against MDR-Enterobacteriaceae, and $A$. baumannii isolates [12], and omadacycline, active against MRSA, MDR-Streptococcus pneumoniae, and ESBL-producing Escherichia coli isolates [13] are briefly presented in Table 1.

Table 1. Current promising antibiotics in treating CRE infections ${ }^{1}$

\begin{tabular}{cccc}
\hline Drug & Mechanism of action & Indications & Limitations \\
\hline Ceftazidime/avibactam & Cell wall synthesis inhibitor & $\begin{array}{c}\text { cUTI, cIAI, BSI, } \\
\text { pneumonia }\end{array}$ & Occurrence of resistance \\
\hline $\begin{array}{c}\text { Ceftolozane/tazobactam } \\
\text { meropenem/varbobacta }\end{array}$ & Inhibition of PBPs & cUT, cIAI & Occurrence of resistance \\
\hline $\begin{array}{c}\text { Ceftaroline/avibactam } \\
\text { cUTI, cIAI, BSI, } \\
\text { pneumonia }\end{array}$ & cUTI & $\begin{array}{c}\text { Occurrence of resistance } \\
\text { due to mutations in } \\
\text { KPC-producing } \\
\text { Enterobacteriaceae }\end{array}$ \\
\hline $\begin{array}{c}\text { Imipenem/cilastatin- } \\
\text { relebactam }\end{array}$ & Renal dehydropeptidase inhibitor/ \\
beta-lactamase inhibitor & cUTI, cIAI, pneumonia & $\begin{array}{c}\text { Severe hypersensitivity } \\
\text { reactions }\end{array}$ \\
\hline $\begin{array}{c}\text { Aztreonam/avibactam } \\
\text { Cell wall synthesis inhibitor }\end{array}$ & Cell wall synthesis inhibitor & cIAI & $\begin{array}{c}\text { Likelihood of resistance } \\
\text { among MBL- and } \\
\text { AmpC-co-producing } \\
\text { K.pneumoniae }\end{array}$ \\
\hline Meropenem/nacubactam & Protein synthesis inhibitor & cUTI, BSI, pneumonia & $\begin{array}{c}\text { Ineffective against MBL- } \\
\text { producers }\end{array}$ \\
\hline
\end{tabular}




\begin{tabular}{|c|c|c|c|}
\hline Eravacycline & Protein synthesis inhibitor & cIAI, pneumonia & $\begin{array}{l}\text { Not indicated for the } \\
\text { treatment of cUTI }\end{array}$ \\
\hline Cefiderocol & Cell wall synthesis inhibitor & cUTI & $\begin{array}{c}\text { Under investigation in } \\
\text { clinical trials }\end{array}$ \\
\hline Omadacycline & Protein synthesis inhibitor & $\begin{array}{l}\text { cUTI, pneumonia, } \\
\text { acute SI }\end{array}$ & $\begin{array}{c}\text { Limited action against } \\
\text { ESBL-producing } K . \\
\text { pneumoniae }\end{array}$ \\
\hline Delafloxacin & $\begin{array}{c}\text { Protein synthesis inhibitor } \\
\text { (topoisomerase IV and DNA } \\
\text { gyrase) }\end{array}$ & Acute SI, pneumonia & $\begin{array}{c}\text { Peripheral neuropathy } \\
\text { and central nervous } \\
\text { system effects }\end{array}$ \\
\hline
\end{tabular}

1 cUTI, complicated urinary tract infections, cIAI, complicated intra-abdominal infections, BSI, bloodstream infections, SI- skin infections, NA- not available.

\section{Future Perspectives in CRE Treatment}

In recent years, the scientific community has focused its efforts on identifying new strategies for combating drug resistance by repositioning non-antibiotic drugs in the antimicrobial arsenal or reconceptualizing old antibiotics.

One of the methodologies in the post-antibiotic era is the use of non-antibiotic drugs for the treatment of multidrug-resistant infections [14]. The benefits are considerable, as the details of these drugs' pharmacokinetics and toxicity are already known, and the drugs can be passed directly into phase 2 of clinical trials [15]. However, the costly disadvantage of clinical trials and patent rights remains [16]. Several drugs recently administered either alone or in conjunction with classical antibiotics have been shown to be effective in removing resistance in CRE, such as antiretroviral compounds (Zidovudine) [17,18], antifungals (Cyclopirox) [19], anticancer compounds (Gallium, Mitotane, Tamoxifen) [20-22], and antidepressants (Sertraline) [23]. Many of these even have different therapeutic targets than conventional antibiotics, which act on DNA, cell wall, or protein translation. Ciclopirox effectively inhibits CRE growth despite resistant status by interfering with galactose metabolism and LPS biosynthesis [19]. Gallium can inhibit ferric redox reactions and associated pathways due to its similarity to iron and can stop the bacterial growth of microorganisms resistant to the last-resort antibiotics [20].

Another promising strategy in the context of antibiotic resistance is the use of liquid ion-based antimicrobial agents (ILs). ILs are generally defined as salts composed solely of cations and anions, with melting points below $100^{\circ} \mathrm{C}$, mostly liquid at room temperature [24]. ILs are not new compounds, dating back more than a century, but have recently come to the scientific community's attention due to the tunable biological properties that allow them to be exploited to generate new and unlimited pharmacological combinations with antimicrobial effects [25]. For example, Ferraz et al. Reported that the administration of ampicillin-based ILs inhibited the bacterial growth of several drug-resistant Gram-negative species (E. coli, Klebsiella pneumoniae), as well Gram-positive strains of Staphylococcus aureus and Enterococcus faecalis, being superior to sodium ampicillin and bromide and chloride salts. Also, the administration of ILs led to the reduction of MIC for two Gram-negative antibiotic-resistant E. coli strains harboring TEM and CTX M9 and CTX M2, respectively, demonstrating the potency of these ampicillin-based ILs for fighting Gram-negative resistant bugs [26]. Other relatively recent studies have shown the effectiveness of combining organic cations such as choline, alkylammonium, alkylpyridiniums, and alkylimidazoliums with various inorganic anions or antibiotics (ampicillinate, carbenicillinate, oxacillinate and cephalothinate or penicillin hydrolyzate, and amoxicillin hydrolyzate) in combating the problem of antibiotic resistance in various Enterobacteriaceae strains [26-29]. ILs can also be combined with phage therapy or lysine therapy to strengthen the therapeutic arsenal in the context of antibiotic resistance [30]. Important attention is also given to polyionic liquids (PILs), which can be designated to achieve amphiphilicity, thereby allowing the polymer's rapid and efficient transfer through the lipid bilayer of the bacterial membrane [31]. It has been documented that PILs with high cation density and long alkyl chains have superior antimicrobial efficacy to their small-molecule counterparts [32]. However, the use of PILs is currently limited by bioaccumulation in the environment, and studies are focused on identifying compounds with optimal biodegradability [31]. 
Testing drugs in clinical trials to investigate their ability to kill bacteria in ways other than conventional ones is extremely laborious and expensive, and most of the time, the results are modest. Thus, the development of new methodologies that reduce costs and increase the discovery of new antibiotics is essential to reinvigorate the antibiotic pipeline. Machine learning approaches can address many of these issues associated with the synthesis, identification, and clinical validation of new compounds with antibiotic properties. A pioneering deep-learning approach has identified new antibiotics from a pool of more than 107 million molecules, also known as the ZINC15 database. Remarkably, one of these compounds, halicin, is structurally divergent from conventional antibiotics and is potent over a wide range of microorganisms, including Mycobacterium tuberculosis and CRE. Halicin efficiency on pan-resistant Acinetobacter baumannii was also confirmed by in vivo studies. This study highlighted the pivotal role of artificial intelligence approaches in describing and predicting potential candidates' properties in reversing antibiotic resistance [33]. Also, relatively recent studies highlight the role of machine learning approaches in optimizing antibiotic combinations to reverse carbapenem resistance in Gram-negative organisms such as A. baumannii [34].

Another strategy exploited to reverse antibiotic resistance comes from synthetic biology and involves redesigning existing antibiotics to overcome natural resistance mechanisms. The concept is based on developing a 'LEGO' set of molecular pieces that can be altered and joined together to generate larger molecules with improved antibiotic capabilities. This has recently been demonstrated with a new class of drugs called streptogramins, which block bacterial growth by interfering with protein synthesis. In this regard, Li et al. built new streptogramins from scratch, creating a series of modules that can be modified as needed to generate a series of variations in the structure of streptogramin molecules. After modifying and assembling these molecular LEGOs, it was observed that these variations have antimicrobial activity on a wide range of pathogens, including streptogramin-resistant $S$. aureus. Therefore, the synthesis and assembly of slightly modified modules can revitalize several antibiotics classes that have been abandoned due to the natural mechanisms of bacterial resistance, offering new hopes in the war against CRE [35]. Another LEGO approach is expected to revitalize endolysins, enzymes exploited by bacteriophages to produce cell lysis and virion release, and currently exploited for the therapeutic management of Gram-negative bacteria [36].

Author Contributions: MCC conceived the manuscript; COV, EGD, and REC drafted the manuscript. IG, IB, and MCC revised the manuscript.

Funding: This study was supported by the Romanian Executive Agency for Higher Education, Research, Development and Innovation (https://uefiscdi.gov.ro/) research projects PN-III-P4-ID-PCCF-2016-0114 POSCCE (RADAR), PN-III-P1-1.1-PD-2016-1798 (PD-148), and PN-III-P1-1.1-PD-2016-2137. The authors declare that they have no known competing financial interests or personal relationships that could have appeared to influence the work reported in this paper. The funding had no role in study design, data collection and analysis, decision to publish, or preparation of the manuscript.

Conflicts of Interest: The authors declare no conflict of interest. The funders had no role in the design of the study; in the collection, analyses, or interpretation of data; in the writing of the manuscript, or in the decision to publish the results.

\section{References}

1. World Health Organization WHO Priority Pathogens List for R\&D of New Antibiotics. 2017.

2. Lee, C.-M.; Lai, C.-C.; Chiang, H.-T.; Lu, M.-C.; Wang, L.-F.; Tsai, T.-L.; Kang, M.-Y.; Jan, Y.-N.; Lo, Y.-T.; Ko, W.-C.; et al. Presence of multidrug-resistant organisms in the residents and environments of longterm care facilities in Taiwan. J. Microbiol. Immunol. Infect. 2017, 50, 133-144, doi:10.1016/j.jmii.2016.12.001.

3. D'Angelo, R.G.; Johnson, J.K.; Bork, J.T.; Heil, E.L. Treatment options for extended-spectrum betalactamase (ESBL) and AmpC-producing bacteria. Expert Opin. Pharmacother. 2016, 17, 953-967, doi:10.1517/14656566.2016.1154538.

4. Centers for Disease Control and Prevention National action plan for combating antibiotic-resistant bacteria. 
2015.

5. Infectious Disease Society of America The 10 x'20 Initiative: pursuing a global commitment to develop 10 new antibacterial drugs by 2020. Clin. Infect. Dis. 2010, 50, 1081-1083, doi:10.1086/652237.

6. D’Onofrio, V.; Conzemius, R.; Varda-Brkić, D.; Bogdan, M.; Grisold, A.; Gyssens, I.C.; Bedenić, B.; Barišić, I. Epidemiology of colistin-resistant, carbapenemase-producing Enterobacteriaceae and Acinetobacter baumannii in Croatia. Infect. Genet. Evol. 2020, 81, 104263, doi:10.1016/j.meegid.2020.104263.

7. Rodríguez-Baño, J.; Gutiérrez-Gutiérrez, B.; Machuca, I.; Pascual, A. Treatment of Infections Caused by Extended-Spectrum-Beta-Lactamase-, AmpC-, and Carbapenemase-Producing Enterobacteriaceae. Clin. Microbiol. Rev. 2018, 31, doi:10.1128/CMR.00079-17.

8. Falagas, M.E.; Karageorgopoulos, D.E.; Nordmann, P. Therapeutic options for infections with Enterobacteriaceae producing carbapenem-hydrolyzing enzymes. Future Microbiol. 2011, 6, 653-666, doi:10.2217/fmb.11.49.

9. Livermore, D.M.; Warner, M.; Mushtaq, S.; Doumith, M.; Zhang, J.; Woodford, N. What remains against carbapenem-resistant Enterobacteriaceae? Evaluation of chloramphenicol, ciprofloxacin, colistin, fosfomycin, minocycline, nitrofurantoin, temocillin and tigecycline. Int. J. Antimicrob. Agents 2011, 37, 415419, doi:10.1016/j.ijantimicag.2011.01.012.

10. Karaiskos, I.; Lagou, S.; Pontikis, K.; Rapti, V.; Poulakou, G. The “Old” and the “New” Antibiotics for MDR Gram-Negative Pathogens: For Whom, When, and How. Front. Public Heal. 2019, 7, 151, doi:10.3389/fpubh.2019.00151.

11. Jean, S.-S.; Gould, I.M.; Lee, W.-S.; Hsueh, P.-R. New Drugs for Multidrug-Resistant Gram-Negative Organisms: Time for Stewardship. Drugs 2019, 79, 705-714, doi:10.1007/s40265-019-01112-1.

12. Kidd, J.M.; Kuti, J.L.; Nicolau, D.P. Novel pharmacotherapy for the treatment of hospital-acquired and ventilator-associated pneumonia caused by resistant gram-negative bacteria. Expert Opin. Pharmacother. 2018, 19, 397-408, doi:10.1080/14656566.2018.1438408.

13. Pfaller, M.A.; Huband, M.D.; Rhomberg, P.R.; Flamm, R.K. Surveillance of Omadacycline Activity against Clinical Isolates from a Global Collection (North America, Europe, Latin America, Asia-Western Pacific), 2010-2011. Antimicrob. Agents Chemother. 2017, 61, doi:10.1128/AAC.00018-17.

14. Peyclit, L.; Baron, S.A.; Rolain, J.-M. Drug Repurposing to Fight Colistin and Carbapenem-Resistant Bacteria. Front. Cell. Infect. Microbiol. 2019, 9, 193, doi:10.3389/fcimb.2019.00193.

15. Kaul, G.; Shukla, M.; Dasgupta, A.; Chopra, S. Update on drug-repurposing: is it useful for tackling antimicrobial resistance? Future Microbiol. 2019, 14, 829-831.

16. Miró-Canturri, A.; Ayerbe-Algaba, R.; Smani, Y. Drug Repurposing for the Treatment of Bacterial and Fungal Infections. Front. Microbiol. 2019, 10, 41, doi:10.3389/fmicb.2019.00041.

17. Hu, Y.; Liu, Y.; Coates, A. Azidothymidine Produces Synergistic Activity in Combination with Colistin against Antibiotic-Resistant Enterobacteriaceae. Antimicrob. Agents Chemother. 2019, 63, doi:10.1128/AAC.01630-18.

18. Ng, S.M.S.; Sioson, J.S.P.; Yap, J.M.; Ng, F.M.; Ching, H.S. V; Teo, J.W.P.; Jureen, R.; Hill, J.; Chia, C.S.B. Repurposing Zidovudine in combination with Tigecycline for treating carbapenem-resistant Enterobacteriaceae infections. Eur. J. Clin. Microbiol. Infect. Dis. 2018, 37, 141-148, doi:10.1007/s10096-0173114-5.

19. Carlson-Banning, K.M.; Chou, A.; Liu, Z.; Hamill, R.J.; Song, Y.; Zechiedrich, L. Toward repurposing ciclopirox as an antibiotic against drug-resistant Acinetobacter baumannii, Escherichia coli, and Klebsiella pneumoniae. PLoS One 2013, 8, e69646, doi:10.1371/journal.pone.0069646. 
20. Hijazi, S.; Visaggio, D.; Pirolo, M.; Frangipani, E.; Bernstein, L.; Visca, P. Antimicrobial Activity of Gallium Compounds on ESKAPE Pathogens. Front. Cell. Infect. Microbiol. 2018, 8, 316, doi:10.3389/fcimb.2018.00316.

21. Tran, T.B.; Wang, J.; Doi, Y.; Velkov, T.; Bergen, P.J.; Li, J. Novel Polymyxin Combination With Antineoplastic Mitotane Improved the Bacterial Killing Against Polymyxin-Resistant MultidrugResistant Gram-Negative Pathogens. Front. Microbiol. 2018, 9, 721, doi:10.3389/fmicb.2018.00721.

22. Hussein, M.H.; Schneider, E.K.; Elliott, A.G.; Han, M.; Reyes-Ortega, F.; Morris, F.; Blastovich, M.A.T.; Jasim, R.; Currie, B.; Mayo, M.; et al. From Breast Cancer to Antimicrobial: Combating Extremely Resistant Gram-Negative “Superbugs” Using Novel Combinations of Polymyxin B with Selective Estrogen Receptor Modulators. Microb. Drug Resist. 2017, 23, 640-650, doi:10.1089/mdr.2016.0196.

23. Otto, R.G.; van Gorp, E.; Kloezen, W.; Meletiadis, J.; van den Berg, S.; Mouton, J.W. An alternative strategy for combination therapy: Interactions between polymyxin B and non-antibiotics. Int. J. Antimicrob. Agents 2019, 53, 34-39, doi:10.1016/j.ijantimicag.2018.09.003.

24. Welton, T. Ionic liquids: a brief history. Biophys. Rev. 2018, 10, 691-706, doi:10.1007/s12551-018-0419-2.

25. Pendleton, J.N.; Gilmore, B.F. The antimicrobial potential of ionic liquids: A source of chemical diversity for infection and biofilm control. Int. J. Antimicrob. Agents 2015, 46, 131-139, doi:10.1016/j.ijantimicag.2015.02.016.

26. Ferraz, R.; Silva, D.; Dias, A.R.; Dias, V.; Santos, M.M.; Pinheiro, L.; Prudêncio, C.; Noronha, J.P.; Petrovski, Ž.; Branco, L.C. Synthesis and Antibacterial Activity of Ionic Liquids and Organic Salts Based on Penicillin G and Amoxicillin hydrolysate Derivatives against Resistant Bacteria. Pharmaceutics 2020, 12, doi:10.3390/pharmaceutics12030221.

27. Cole, M.R.; Hobden, J.A.; Warner, I.M. Recycling antibiotics into GUMBOS: a new combination strategy to combat multi-drug-resistant bacteria. Molecules 2015, 20, 6466-6487, doi:10.3390/molecules20046466.

28. Dang, T.; Nizamov, I.S.; Salikhov, R.Z.; Sabirzyanova, L.R.; Vorobev, V. V; Burganova, T.I.; Shaidoullina, M.M.; Batyeva, E.S.; Cherkasov, R.A.; Abdullin, T.I. Synthesis and characterization of pyridoxine, nicotine and nicotinamide salts of dithiophosphoric acids as antibacterial agents against resistant wound infection. Bioorg. Med. Chem. 2019, 27, 100-109, doi:10.1016/j.bmc.2018.11.017.

29. Ferraz Ricardo A4 - Teixeira, Vânia A4 - Rodrigues, Débora A4 - Fernandes, Rúben A4 - Prudêncio, Cristina A4 - Noronha, João Paulo A4 - Petrovski, Željko A4 - Branco, Luís C., R.A.-F. Antibacterial activity of Ionic Liquids based on ampicillin against resistant bacteria. RSC Adv. 2013, v. 4, 4301-4307-2013 v.4 no.9, doi:10.1039/c3ra44286a.

30. Prudêncio, C.; Vieira, M.; Van der Auweraer, S.; Ferraz, R. Recycling Old Antibiotics with Ionic Liquids. Antibiot. (Basel, Switzerland) 2020, 9, doi:10.3390/antibiotics9090578.

31. Smith, C.A.; Cataldo, V.A.; Dimke, T.; Stephan, I.; Guterman, R. Antibacterial and Degradable Thioimidazolium Poly(ionic liquid). ACS Sustain. Chem. Eng. 2020, 8, 8419-8424, doi:10.1021/acssuschemeng.0c02666.

32. Zheng, Z.; Xu, Q.; Guo, J.; Qin, J.; Mao, H.; Wang, B.; Yan, F. Structure-Antibacterial Activity Relationships of Imidazolium-Type Ionic Liquid Monomers, Poly(ionic liquids) and Poly(ionic liquid) Membranes: Effect of Alkyl Chain Length and Cations. ACS Appl. Mater. Interfaces 2016, 8, 12684-12692, doi:10.1021/acsami.6b03391.

33. Stokes, J.M.; Yang, K.; Swanson, K.; Jin, W.; Cubillos-Ruiz, A.; Donghia, N.M.; MacNair, C.R.; French, S.; Carfrae, L.A.; Bloom-Ackermann, Z.; et al. A Deep Learning Approach to Antibiotic Discovery. Cell 2020, 180, 688-702.e13, doi:10.1016/j.cell.2020.01.021.

34. Smith, N.M.; Lenhard, J.R.; Boissonneault, K.R.; Landersdorfer, C.B.; Bulitta, J.B.; Holden, P.N.; Forrest, A.; 
Nation, R.L.; Li, J.; Tsuji, B.T. Using machine learning to optimize antibiotic combinations: dosing strategies for meropenem and polymyxin B against carbapenem-resistant Acinetobacter baumannii. Clin. Microbiol. Infect. 2020, doi:10.1016/j.cmi.2020.02.004.

35. Li, Q.; Pellegrino, J.; Lee, D.J.; Tran, A.A.; Chaires, H.A.; Wang, R.; Park, J.E.; Ji, K.; Chow, D.; Zhang, N.; et al. Synthetic group A streptogramin antibiotics that overcome Vat resistance. Nature 2020, 586, 145-150, doi:10.1038/s41586-020-2761-3.

36. Gerstmans, H.; Criel, B.; Briers, Y. Synthetic biology of modular endolysins. Biotechnol. Adv. 2018, 36, 624640, doi:10.1016/j.biotechadv.2017.12.009.

Publisher's Note: MDPI stays neutral with regard to jurisdictional claims in published maps and institutional affiliations.

(C) 2020 by the authors. Submitted for possible open access publication under the terms and conditions of the Creative Commons Attribution (CC BY) license (http://creativecommons.org/licenses/by/4.0/). 